\title{
Spontaneous Intramural Hematoma of the Jejunum Induced by Anticoagulant Therapy
}

\author{
Takashi Hirosawa, Katsuyoshi Kudoh, Takeshi Aoki, Shinobu Ohnuma, \\ Naoki Tanaka, Hiroaki Musha, Fuyuhiko Motoi, Takashi Kamei, \\ Takeshi Naitoh and Michiaki Unno \\ Department of Surgery, Tohoku University Graduate School of Medicine
}

\begin{abstract}
A spontaneous, intramural, small-bowel hematoma requiring a surgery is a rare complication of anticoagulant therapy. We present a case of an 82 -yearold man who developed abdominal pain in the setting of abnormal coagulation function related to warfarin therapy used as chronic prophylaxis against recurrent pulmonary embolism. Computed tomography (CT) showed wall thickening and luminal narrowing of the jejunum. Dilation of the small bowel proximal to the thickening was also present, indicative of small bowel obstruction. Initially, the patient was treated conservatively, but he later required laparotomy due to worsening of his general condition. A $50 \mathrm{~cm}$ jejunal segment was resected in order to relieve the intestinal obstruction and to arrest the bleeding. For intramural, small-bowel hematoma, conservative medical management should be the first treatment of choice, but surgical intervention may be indicated if conservative treatment is not successful.
\end{abstract}

Key words: Anticoagulant, Intramural hematoma, Surgical treatment

\section{Introduction}

Oral anticoagulants are widely prescribed in many conditions as prophylactic and therapeutic agents. Warfarin is the most frequently used of these drugs,

Received: August 17, 2017/ Accepted: December 6, 2017 Correspondence to: Kudoh Katsuyoshi

Department of Surgery, Tohoku University Graduate School of Medicine

1-1 Seiryo-machi, Aoba-ku, Sendai, Miyagi, 980-8574, Japan and its most serious complication is excessive anticoagulation, with bleeding; such spontaneous bleeding occurs in about 7.6 per 100 patient-years and primarily in the digestive tract ${ }^{1)}$.

In Switzerland, incidence of small bowel hematomas was reported to be 1: 2,500 patients on anticoagulation per year ${ }^{2}$. However, there have not been many cases that needed surgical treatment ${ }^{3}$.

We present a case of spontaneous intramural hematoma of the jejunum which was presumed to have occurred due to drug interaction during warfarin anti-coagulant therapy.

\section{Case Report}

A man in his 80 's with a history several years previously of pulmonary embolism and chronic obstructive pulmonary disease was admitted for radiation therapy for lung carcinoma. His medications included allopurinol $100 \mathrm{mg} /$ day, warfarin $3 \mathrm{mg} /$ day, aspirin $100 \mathrm{mg} /$ day, candesartan cilexetil $8 \mathrm{mg} /$ day and clarithromycin $200 \mathrm{mg} /$ day. Three days after admission, the patient developed abdominal pain and vomiting. Plain and contrast-enhanced computed tomography (CT) demonstrated circumferential wall thickening, luminal narrowing, and intramural hyperdensity of the jejunum with hyperdense ascites in Douglas' pouch (Fig. 1 ). Laboratory investigations showed a hemoglobin level of $11.5 \mathrm{~g} / \mathrm{dl}$, a platelet count of 196 $\times 10^{3} / \mu \mathrm{l}$, a markedly prolonged prothrombin timeinternational normalized ratio (PT-INR) $>7.0$ and a prolonged activated partial thromboplastin time of $72.4 \mathrm{~s}$. Warfarin and aspirin were stopped and replaced with low molecular weight heparin (dalteparin sodium). The patient was treated conservatively with bowel rest, intravenous fluid hydration and in- 


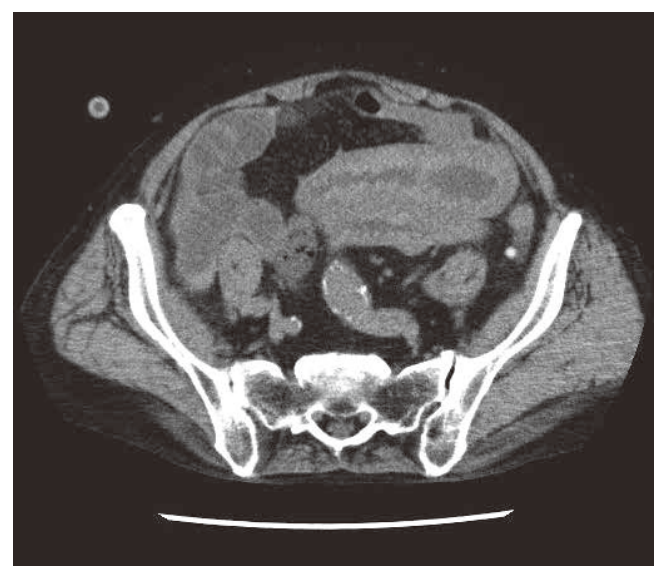

Fig. 1 Abdominal plain CT demonstrating wall thickening, luminal narrowing and hyperdensity of the jejunum.

Dilation of the small bowel proximal to the thickening was also recognized, suggestive of a small bowel obstruction.

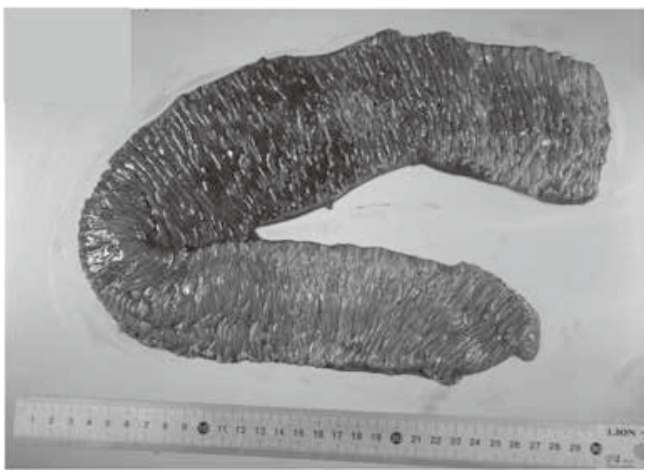

Fig. 2 Resected specimen showing segmental intramural hematoma localized to a part of the jejunum with intact mucosa.

travenous vitamin K. Consequently, the PT-INR improved to 1.39 .

Because of decreasing blood pressure and worsening of the anemia, the patient underwent exploratory laparotomy on suspicion of an exacerbation of anticoagulant-induced intraabdominal hemorrhage. The peritoneal cavity contained about $300 \mathrm{ml}$ of bloody fluid and an approximately $20 \mathrm{~cm}$ segment of the jejunum was markedly thickened and dark-red in color (Fig. 2 ). The adjacent mesentery was also thickened with hematoma but there was no evidence of active bleeding from the mesenteric vessel. In order to relieve the intestinal obstruction and be-

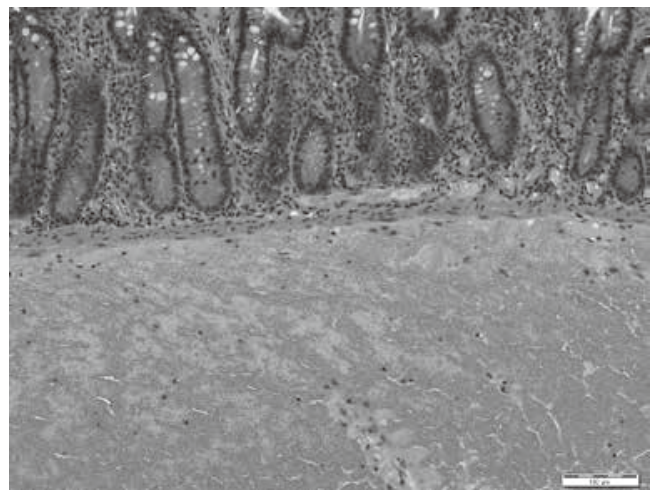

Fig. 3 Pathological examination showing submucosal hemorrhage and intact mucosa and the muscularis mucosa of the jejunum.

cause of concerns of bowel ischemia, partial resection of about $50 \mathrm{~cm}$ of the small intestine was performed. Histopathologic examination showed extensive submucosal hemorrhage of the jejunum without thrombus or necrosis (Fig. 3 ).

His postoperative course was complicated by respiratory failure but he eventually recovered. $\mathrm{He}$ was discharged on the 56th postoperative day with oral intake of rivaroxaban $10 \mathrm{mg}$ daily.

\section{Discussion}

Small bowel intramural hematomas found on autopsy were first reported in 1838 by McLauchlan who described them as "false aneurysmal tumor" occluding the duodenum ${ }^{4}$. The jejunum is the most common site of spontaneous intramural small bowel hematoma, followed by the ileum, and duodenum, whereas traumatic small bowel hematoma commonly affects the duodenum ${ }^{5,6}$.

Over-anticoagulation with warfarin is the most common cause of spontaneous intramural smallbowel hematoma, while unfractionated heparin accounts for few cases $^{7,8)}$. Other aetiological factors ${ }^{5)}$ include hemophilia, idiopathic thrombocytopenic purpura, von Willebrand's disease ${ }^{9)}$, Henoch-Schonlein purpura ${ }^{10)}$, malignancies, and vasculitis. Small bowel hematomas are also associated with pancreatitis and pancreatic carcinoma ${ }^{11)}$. It is most likely that there is a rupture of the terminal artery that branches from the mesentery and penetrates into the muscular layer of the intestinal wall. Therefore, bleeding dissects the intestinal wall between the muscularis mucosa and muscular layers ${ }^{12,13)}$. Pro- 
Spontaneous Intramural Hematoma of the Jejunum Induced by Anticoagulant Therapy

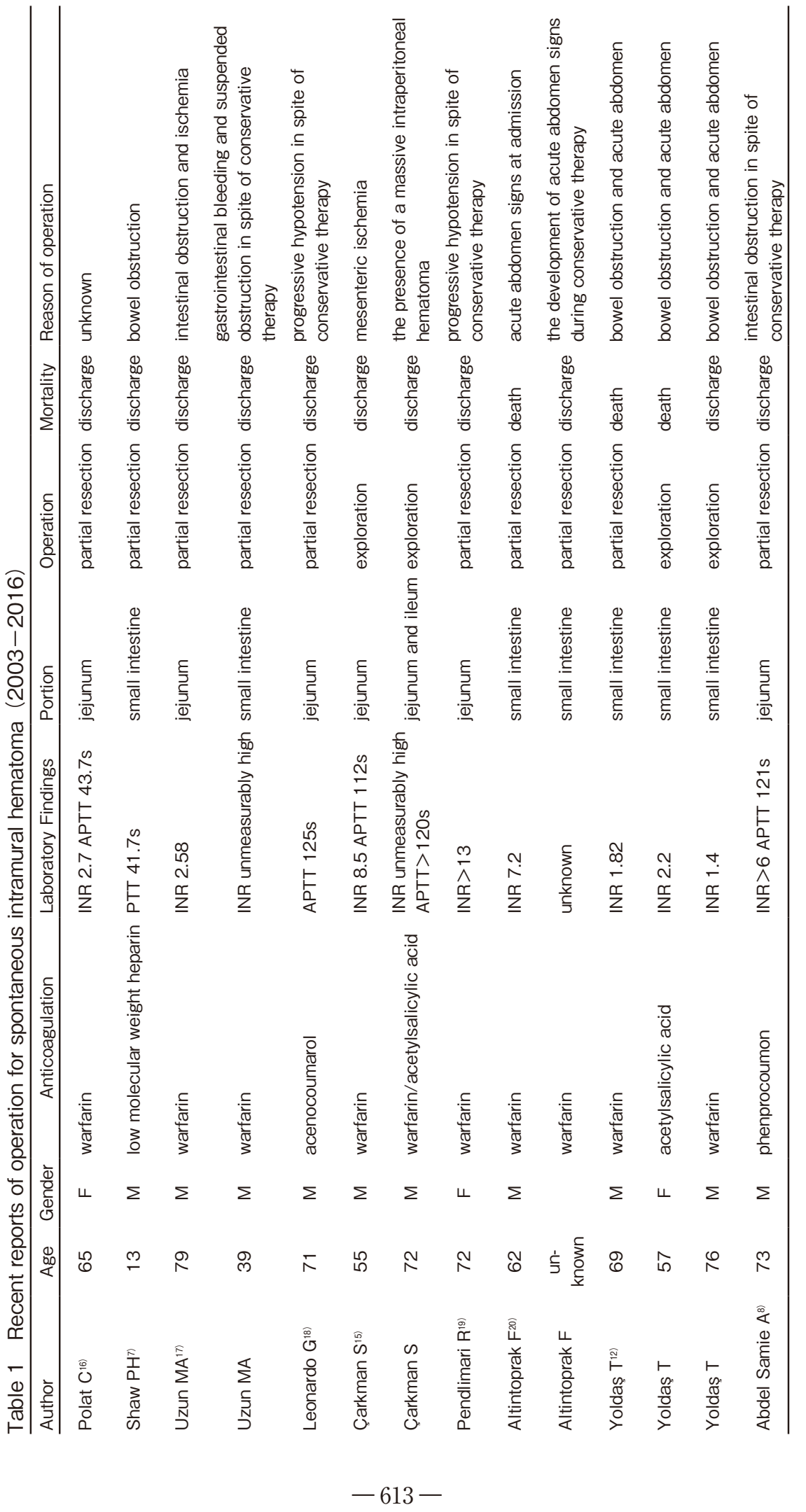


gression of symptoms is due to an intramural osmotic gradient caused by the liquefying hematoma, which causes thickening of the intestinal wall and decreasing the intestinal luminal dimeter by drawing fluid from the surrounding tissues ${ }^{14)}$.

On admission, almost all patients have abdominal pain, and about one half of the patients have nausea and vomiting, which are related to high obstructions involving the duodenum and proximal jejunum. Hemorrhage in the digestive tract is reported in up to $40 \%$ of cases, exteriorized in the form of melena, hematemesis and hematochezia ${ }^{3)}$.

In the presence of signs of peritoneal irritation, hematoma complications like necrosis, perforation and hemoperitoneum should be suspected ${ }^{12}$.

Most patients have an elevated PT-INR, values of which greater than 3 are associated with an increased risk of bleeding ${ }^{15)}$. Hemoglobin levels at presentation are often normal or borderline anemic. Bleeding could also occur even though the coagulation parameters are within the therapeutic range ${ }^{16)}$.

CT is the most useful diagnostic modality of choice, which is sufficiently sensitive and beneficial to diagnose and follow intramural hematoma. Characteristic findings are circumferential bowel wall thickening, intramural hyperdensity, luminal narrowing and intestinal obstruction ${ }^{5}$.

Initially, noncontrast CT should be performed before oral and intravenous contrast medium application, as contrast-enhanced $\mathrm{CT}$ alone may mask the presence of intramural hemorrhage ${ }^{3)}$.

Owing to the rarity of this entity, there is no standardized treatment. However, most reports have recommended conservative therapy as the mainstay of therapy, including bowel rest, nasogastric decompression, correction of electrolyte disturbances, immediate suspension of oral anticoagulants, correction of abnormal coagulation parameters by administering vitamin $\mathrm{K}$, fresh frozen plasma (FFP) and blood transfusion ${ }^{3,12,15)}$. The resolution of a hematoma might start as early as 1 week after the onset and complete resolution usually occurs within 2 months ${ }^{5}$.

Surgical intervention should be considered in patients with significant intramural hemorrhage, suspected ischemia, perforation, peritonitis, or intestinal obstruction that does not resolve with conservative treatment ${ }^{3,12,16)}$. In the present case, we decided to resect the damaged segment, since it was necessary to prevent the worsening general condition of the patient.

The PubMed were used in order to review the literature describing the case who had underwent surgical treatment by a diagnosis of intramural hematoma of small intestine from 2003 to 2016 (Table $\mathbf{1}$, 14cases). More seriously ill patients should tend to have surgical indication, however, recent studies present acceptably good prognosis of patients who underwent surgical therapy (out of 14 patients, 11 were discharged alive) $)^{7,8,12,15-20)}$.

In our case, it is speculated that the main cause the onset was drug interaction with warfarin. The potential for warfarin to adversely interact with other drugs including antiplatelet agents, antibiotics and agents against gout, causing fluctuations in the anticoagulant effect, is widely recognized ${ }^{21)}$. This patient had taken multiple medications and aspirin, clarithromycin and allopurinol, which had the possibility of augmenting excessive effect of warfarin. On the other hand, polypharmacy is a major problem in the elderly population and increases the chance of drug interaction ${ }^{19)}$. We presume that ruptures of the terminal artery in the intestinal wall occasionally happen, but that the bleeding would stop if the coagulant function is normal. However, abnormal anticoagulation by excessive effect of warfarin causes an augmentation of intramural hematoma.

In conclusion, although intramural small bowel hematoma requiring a surgery is a rare complication of anticoagulant therapy, the possibility of this entity in patients presenting with acute abdominal pain should be considered for an accurate diagnosis and treatment. We should consider a surgical procedure should be indicated if conservative treatment is not successful or if there is the worsening of general condition.

Conflict of interest: None.

\section{References}

1) Palareti G, Leali N, Coccheri S, et al : Bleeding complications of oral anticoagulant treatment: an inception-cohort, prospective collaborative study (ISCOAT). Italian Study on Complications of Oral Anticoagulant Therapy. Lancet 348:423-428, 1996

2) Bettler S, Montani S, Bachmann F : Incidence of intramural digestive system hematoma in anticoagulation. Epidemiologic study and clinical aspects of 


\section{Spontaneous Intramural Hematoma of the Jejunum Induced by Anticoagulant Therapy}

59 cases observed in Switzerland(1970-1975). Schweiz Med Wochenschr $113: 630-636,1983$

3) Sorbello MP, Utiyama EM, Parreira JG, et al : Spontaneous intramural small bowel hematoma induced by anticoagulant therapy : review and case report. Clinics $62: 785-790,2007$

4) McLauchlan J : Fatal false aneurysmal tumour occupying nearly the whole of the duodenum. Lancet $2: 203-205,1883$

5) Abbas MA, Collins JM, Olden KW : Spontaneous intramural small-bowel hematoma:imaging findings and outcome. AJR Am J Roentgenol 179 :13891394, 2002

6) Hughes CE 3rd, Conn J Jr, Sherman JO : Intramural hematoma of the gastrointestinal tract. Am J Surg $133: 276-279,1977$

7) Shaw PH, Ranganathan S, Gaines B : A spontaneous intramural hematoma of the bowel presenting as obstruction in a child receiving low-molecular-weight heparin. J Pediatr Hematol Oncol 27:558-560, 2005

8) Abdel SA, Sun R, Huber A, et al : Spontaneous intramural small-bowel hematoma secondary to anticoagulant therapy : a case series. Med Klin Intensivmed Notfmed $108:$ 144-148, 2013

9) Eichele DD, Ross M, Tang P, et al : Spontaneous intramural duodenal hematoma in type $2 \mathrm{~B}$ von Willebrand disease. World J Gastroenterol 19 :72057208, 2013

10) Sutherland GA : Intussusception and Henoch's purpura. Br J Dis Child 1:23-28, 1904

11) Dubois J, Guy F, Porcheron J:A pancreatic-indued intramural duodenal hematoma : a case report and literature review. Hepatogastroenterology 50 : 1689-1692, 2003
12) Yoldaş T, Erol V, Çalışkan C, et al : Spontaneous intestinal intramural hematoma: What to do and not to do. Ulus Cerrahi Derg $29: 72-75,2013$

13) Hale JE : Intramural intestinal haemorrhage : a complication of anticoagulant therapy. Postgrad Med J $51: 107-110,1975$

14) Abbas MA, Collins JM, Olden KW : Spontaneous intramural small-bowel hematoma: clinical presentation and long-term outcome. Arch Surg 137:306310,2002

15) Çarkman S, Özben V, Saribeyoğlu K, et al : Spontaneous intramural hematoma of the small intestine. Ulus Travma Acil Cerrahi Derg $16: 165-169,2010$

16) Polat C, Dervisoglu A, Guven H, et al : Anticoagulant-induced intramural intestinal hematoma. Am J Emerg Med 21:208-211, 2003

17) Uzun MA, Koksal N, Gunerhan Y, et al : Intestinal obstruction due to spontaneous intramural hematoma of the small intestine during warfarin use : a report of two cases. Eur J Emerg Med 14:272-273, 2007

18) Leonardo G, Catracchia V, Molina A : Spontaneous intestinal hematoma as anticoagulant treatment complication. G Chir $29: 421-423,2008$

19) Pendlimari R, Anaparthy R, Sugumar A : Drug interaction presenting as acute abdomen. World J Gastrointest Pharmacol Ther 1:40-42, 2010

20) Altintoprak F, Dikıcıer E, Akyüz M : A retrospective review of patients with non-traumatic spontaneous intramural hematoma. Turk J Gastroenterol $24: 392-399,2013$

21) Wells PS, Holbrook AM, Crowther NR, et al : Interactions of warfarin with drugs and food. Ann Intern Med $121: 676-683,1994$ 